\title{
El despliegue de la política habitacional "Conformación de Consejos de Administración" en el barrio 7 de
Septiembre. Rosario, Argentina
}

The deployment of the housing policy "Building Administrative Councils" in the 7 September neighborhood. Rosario, Argentina

CARDOSO ADUR ANDREA* UBERTI ORNELLA** ZAMBONI VIRGINIA***

*Tesista en Lic. En Ciencia Política. Correo electrónico: ajcardosoadur@gmail.com

**Lic. en Ciencia Política. Docente alumna en Tecnologías de la Administración Pública de la Universidad Nacional de Rosario. Correo electrónico: orlyuberti@gmail.com

${ }^{* * *}$ Tesista en Lic. en Ciencia Política. Docente alumna de Estructura Social Argentina de la Universidad Nacional de Rosario. Correo electrónico: virginiaz2901@gmail.com

\section{Resumen}

En este trabajo nos proponemos realizar un análisis del diseño, implementación y control de la política pública referida a la Conformación de Consejos de Administración, para organizar vecinos, en los Complejos Habitacionales de las periferias de la ciudad de Rosario, Argentina.

Rosario es una de las ciudades más pobladas de la Argentina, en la cual se albergan muchos de los complejos sociales urbanísticos con mayor capacidad de la región, resultado de las políticas habitacionales asistencialistas frente al déficit habitacional creciente de los años' 70 . Estos complejos fueron

\section{Abstract}

In this research we propose an analysis of the design, implementation and results of the public policy Building Administrative Councils, which is supposed to organize neighbors in the housing areas at the outskirts of the city.

Rosario is one of the most populated cities in Argentina and home to many of the largest housing areas in the region. The latter is a result of the housing assistance policies that tried to deal with the growing housing deficit in the 1970s. These housing areas were abandoned by the State for two decades and, as a consequence, this left a great number of unresolved 
abandonados por el Estado por dos décadas, tanto en los 80 como en los '90, lo que ha causado que se fueran apilando problemáticas que nunca fueron resueltas. Enfocaremos nuestro análisis en el Barrio 7 de Septiembre con el objetivo de dilucidar si esta política propuesta por oficinas estatales logra organizar a los vecinos.

\section{Palabras clave}

Consejos de Administración Políticas Públicas - Convivencia issues. Among these housing areas there is the neighborhood of 7 de Septiembre. In this context, our analysis will focus on this neighborhood and will try to find out whether this policy adopted by Government Offices achieve the organization of the neighbors.

\section{Keywords}

Administrative Councils - Public Policies - Coexistence

\section{Introducción}

En este trabajo nos proponemos realizar un análisis del despliegue de la política pública referida a la Conformación de Consejos de Administración en los Complejos Habitacionales Fo.Na.Vi. ${ }^{1}$ en el barrio 7 de Septiembre, Rosario, en el período 20132016.

La cuestión acerca de la re-habilitación de los Complejos Habitacionales tanto a niveles convivenciales como estructurales ingresó a las agendas de gestión por medio de la Ley Provincial $N^{\circ} 12.953$ del año 2008, pero la política de conformación de Consejos de Administración en los Consorcios de los Complejos Fo.Na.VI, más allá de formar parte de dicha ley, recién adquirió impulso a partir del lanzamiento del Plan Abre. ${ }^{2}$

El mismo fue puesto en marcha desde diciembre de 2013 con el propósito de integrar las diversas estrategias de intervención que desde el estado provincial se venían llevando adelante para recuperar vínculos sociales y mejorar la calidad de vida en barrios de distintas ciudades de la provincia. De esta manera, el Plan Abre incorpora en uno de sus ejes la conformación de Consejos de Administración en

\footnotetext{
1 Fo.Na.Vi hace referencia al Fondo Nacional a la Vivienda, constituido con el objetivo de destinar recursos para la construcción de viviendas sociales. Con el avance de los gobiernos neoliberales en la década del '90 estos pasaron a ser responsabilidad de las administraciones provinciales, las que, dada la imposibilidad de hacerse cargo de los mismos, fomentaron indirectamente su decaimiento y abandono.

${ }^{2}$ El Plan Abre es un programa de intervención integral sustentado en la coordinación entre diversas áreas que conforman el Gabinete Social de la provincia de Santa Fe y gobiernos locales, con el fin de recuperar los vínculos sociales en barrios de las principales ciudades de la Provincia. Esta propuesta se puso en marcha en diciembre de 2013 abarcando las ciudades Santa Fe, Rosario y Villa Gobernador Gálvez, y posteriormente se sumaron las ciudades de Santo Tomé, y Pérez.
} 
estos complejos habitacionales, brindándole a esta última un marco más integral y coordinado de acciones. En este sentido, nos surgieron interrogantes respecto a los cambios y resignificaciones que se dieron en la política con su incorporación al Plan Abre.

Abordamos el caso de los Complejos Habitacionales del barrio 7 de Septiembre con el objetivo de analizar el despliegue de la política en un territorio concreto.

El barrio 7 de Septiembre es el barrio en que mayor alcance tuvo la implementación de la política que nos interesa analizar. La política fue bien recibida por los beneficiarios y obtuvo un buen resultado en términos comparativos respecto a otros barrios $^{3}$ : en el recorte temporal que tomamos se han logrado conformar casi la totalidad de los Consejos de Administración y la mayoría de los mismos continúan funcionando.

En nuestro análisis descubrimos algunas particularidades del despliegue de la política, en las cuales nos enfocaremos a lo largo de nuestro trabajo. En primer lugar, se abordará lo referido a la historicidad de la cuestión, donde observamos una resignificación de la política por parte de los actores decisores. En relación a ello nos preguntamos ¿cuáles son los motivos del cambio en la toma de posición del Estado frente a la cuestión? y ¿cómo afecta al despliegue de la política su incorporación al Plan Abre? Por otra parte, en el despliegue de la política analizada se ponen en constante tensión los campos público-privado, interpelando qué responsabilidades se otorgan a los actores públicos y privados que intervienen. El interrogante que nos planteamos es entonces ¿por qué el Estado Provincial debe responsabilizarse (o elige responsabilizarse) de organizar a los privados? ¿Cuánto incide en el despliegue de la política el hecho de que la organización de los vecinos revista un carácter privado? Por último, siendo que se trata de una política que involucra en su despliegue a los vecinos que habitan en los Complejos Habitacionales Fo.Na.Vi.s, nos preguntamos si efectivamente se cumplen los objetivos relacionados a la participación ciudadana de la política en este territorio, y cuál es la importancia de esta participación para el despliegue de la política.

\section{Marco conceptual}

Para conceptualizar el despliegue de la política pública consideramos fundamental comenzar enunciando qué entendemos por política pública. Siguiendo a Subirats (1989, p. 41), se trata de una "decisión formal" caracterizada por una conducta o actuación consistente y repetida por parte de aquellos que la llevan a cabo y de aquellos que resultan afectados por la misma. En verdad, dirá el autor, es más que una decisión: es decidir que existe un problema, que dicho problema debe resolverse y cuál es la mejor forma de proceder para ello. De este modo, las políticas públicas suponen un proceso de decisión que se acompaña con una actuación.

\footnotetext{
${ }^{3}$ Hacemos referencia a barrios de la zona sur de Rosario, que se encuentran atravesando graves situaciones de violencia. El ingreso, permanencia y ejecución de la Conformación de Consorcios se vio altamente dificultada.
} 
En la literatura sobre políticas públicas suele hacerse la distinción analítica entre las fases de formulación, implementación y evaluación de las políticas públicas. A nuestro entender, ambas presentan un elevado grado de interdependencia y es por ello que nos interesa analizar el despliegue de la política, entendiendo que se trata de un proceso complejo en el que se formula, se pone en marcha y funciona una política por medio de la interacción de diversos actores con intereses, objetivos y estrategias diferenciados. De este modo hablamos de despliegue para referir al proceso transversal a las diversas fases de la política pública.

Coincidimos con Oszlack y O’Donnell (1976) en que, si bien los actores públicos en sus diversas formas tienen roles claves, nunca son exclusivos en lo que refiere a políticas públicas. El espacio estatal ofrece un poder diferencial en tanto que las acciones que de él surgen tienen una repercusión más profunda y cuentan con respaldo institucional, normativo y coactivo. No obstante, centrar la mirada en los actores públicos puede implicar un sesgamiento sobre el proceso que invisibiliza a otros actores que pueden resultar relevantes a la hora de analizar la política pública. En la actualidad, cada vez son más los actores locales que adquieren relevancia, interviniendo y decidiendo en materia de política pública. En este sentido, los actores locales se autonomizan de la autoridad central y al poner en juego elementos de sus propias racionalidades en su actuación, se generan influencias, negociaciones y ajustes susceptibles de modificar los objetivos iniciales. Lo mismo sucede con la reacción y el accionar de los actores afectados por la política.

\section{Metodología}

En cuanto a lo metodológico consideramos apropiado abordar el análisis sobre la implementación de esta política desde un enfoque de tipo "bottom up". En términos de Morata (1991, p. 162) este tipo de análisis se centra en los aspectos prácticos de la ejecución: la multiplicidad de los actores y de las organizaciones implicados y la variedad de los lazos que los unen; los sistemas de valores, los intereses representados, la autonomía de decisión y el poder político de que disponen, $y$, finalmente, los mecanismos de interacción utilizados y, en especial, la negociación y el intercambio político. Si bien el autor propone este enfoque para el análisis de la fase de implementación, nos resulta útil rescatar algunas de sus herramientas al momento de centrarnos en los aspectos prácticos del despliegue de la política en el territorio abordado.

Consideramos este enfoque puesto que el mismo permite integrar el pluralismo institucional y territorial que presenta todo proceso de decisión y ejecución de políticas públicas, al tiempo que supera algunos presupuestos del enfoque tradicional como la distinción entre "decisión" y "ejecución" o la caracterización de las políticas públicas como procesos lineales que son resultado de una voluntad unitaria. El enfoque "bottom up" se corresponde en mejor medida con nuestra definición de las políticas públicas y de la implementación en tanto entiende que el proceso de toma de decisiones públicas es resultado de las interacciones que se dan entre actores con diferentes intereses, recursos y objetivos (Morata, 1991). Es 
por ello que esta metodología nos permite hacer jugar las fases de diseño e implementación en el análisis del despliegue de la política.

Se trabajó con un mapeo de actores claves, consistente no sólo en sacar un listado de posibles actores presentes en un territorio, sino en conocer sus acciones y objetivos de participación. Entendemos por actores claves aquellos individuos, grupos o instituciones que son afectados o afectan el desarrollo de determinadas actividades, aquellos que poseen información, recursos, experiencia y alguna forma de poder para influenciar la acción de otros (Tapella, 2007, p. 3). El énfasis estuvo puesto en el análisis actoral, identificando los roles (qué función desempeña cada actor y qué objetivo persigue con su accionar), los niveles de poder (qué capacidad tienen de influenciar limitando o facilitando las acciones en pos del despliegue de la política), los posicionamientos frente a la política (interés en favor, en contra o indiferente) y los tipos de relaciones predominantes (fuerte coordinación, débil o nula coordinación, conflicto), entendiendo que estas cuestiones entran en juego en el despliegue de la política.

Se utilizaron fuentes primarias y secundarias relacionadas con la temática ligada al caso elegido. Nos remitimos a leyes provinciales, decretos ejecutivos y planes provinciales y municipales que fundamentan el despliegue de esta política y recurrimos a fuentes de información de tipo periodística, propagandística e informes en páginas oficiales. Por otro lado, realizamos entrevistas no estructuradas a directivos de la Dirección Provincial de Vivienda y Urbanismo (de ahora en adelante -DPVyU-) y, aparte del personal de la Unidad Ejecutora, a responsables de 5 Consejos de Administración, a vecinos habitantes de los Complejos Habitacionales, a funcionarios de la Municipalidad de Rosario y al director de la Vecinal 7 de Septiembre.

A partir de estos instrumentos metodológicos y del acercamiento al territorio, relevamos el funcionamiento de los consorcios al interior del Barrio 7 de Septiembre, analizando no sólo su accionar cotidiano, sino también su interrelación con los diferentes actores presentes en el territorio, incluyendo tanto a las instituciones que han formado parte como a los vecinos beneficiarios de la política. Esto nos permitió conocer el territorio, abordado sus principales problemáticas, las perspectivas y percepciones de los diversos actores involucrados, sus objetivos y lógicas de intervención, así como los vínculos que entre los mismos se establecen.

\section{Una descripción del territorio y sus actores}

La ciudad de Rosario cuenta con nueve complejos habitacionales de tipo FO.NA.Vi a los largo de su extensión. Imitando el patrón de la escala nacional, los mismos rompen con la trama urbana, lo que produce que las relaciones entre los monoblocks y el resto de la ciudad sea dificultosa (Topini y Uberti, 2014). Como puede verse en la imagen de abajo, están en su mayor parte localizados en las periferias de la ciudad, lo que sumado a sus estructuras cerradas le imprime a estos complejos características segregatorias.

El complejo habitacional que será nuestro objeto de análisis es el ubicado en el Barrio 7 de Septiembre. Este barrio, localizado en el distrito noroeste de la ciudad de 
Rosario, cuenta con más de 35.000 habitantes. Es un barrio joven, ya que tiene apenas 35 años, y presenta una enorme diversidad de habitantes. Fue originariamente un barrio metalúrgico dado que las primeras viviendas fueron construidas por la Unión de Obreros Metalúrgicos (UOM). No obstante, a lo largo de sus pocos años de historia el barrio fue cambiando su configuración. Ya no es exclusivamente un barrio metalúrgico: hay comerciantes, empleados públicos, trabajadores, entre otros oficios y profesiones.

Mapa N 1: Ubicación de los Complejos Sociales Habitacionales en la Ciudad de Rosario.

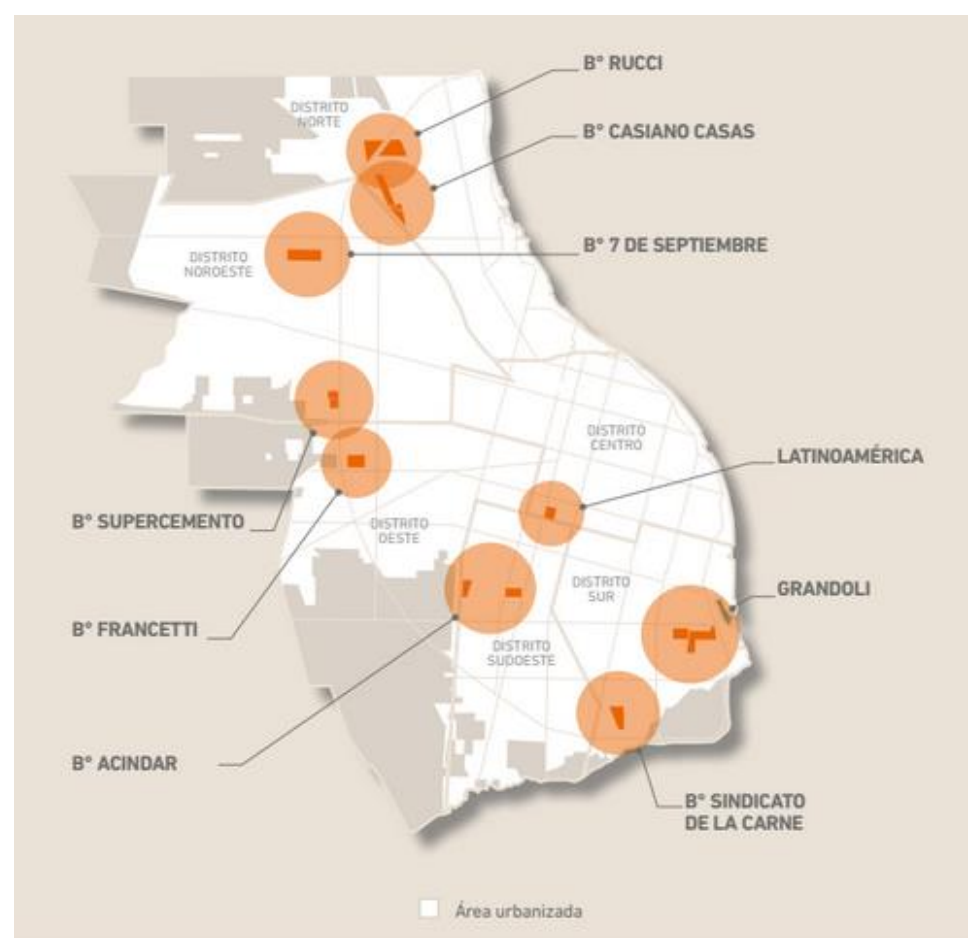

Fuente: Informe de Gestión (2011-2015) de la Municipalidad de Rosario

La cuestión de la propiedad de las tierras ha sido un eje problemático del barrio desde su origen: en primer lugar, fueron propiedad de la UOM. La misma, hacia la década del setenta vendió los lotes al Banco Hipotecario, situación que generó enormes conflictos entre los propietarios y los usuarios de las viviendas. Para la década del ochenta, la DPVyU pasó a tener jurisdicción sobre esas viviendas.

Es importante señalar que el barrio cuenta con cuatro diferentes planes de vivienda, siendo éstos el $94,131,132$ y 210 . Los planes son números que reflejan el momento de entrega de un determinado conjunto de viviendas, por lo que podría decirse que las construcciones en este barrio no se realizaron o terminaron en simultaneidad. Otra de las características es la diferencia entre las viviendas construidas en cada plan; por ejemplo, dentro del plan 132 se encuentran los duplex (casas que comparten medianera) y monoblocks que cuentan con 48 viviendas, mientras que el plan 210 consta de tiras de ocho o dieciséis viviendas cada una. 
Este barrio empezó a mostrar sus fisuras llegado el primer decenio del 2000. Problemas estructurales, de mantenimiento general, tanto en los edificios como en los espacios públicos, empezaron a llamar la atención de medios de comunicación y vecinos. En parte, dichas fisuras son el saldo del modelo económico neoliberal implementado en la década del noventa. La política de ajuste, achique presupuestario y medidas del mercado laboral que provocaron la caída en las tasas de empleo, el aumento del empleo informal, el deterioro de los ingresos y el aumento de los trabajadores en negro, impactó claramente en las condiciones de vida de los habitantes de los monoblocks. Los barrios periféricos de la ciudad se conformaron como nichos de economías delictivas, y el escenario habitual permitía ver en los monoblocks lo putrefacto de sus esquinas, el abandono de sus estructuras y la humedad de sus paredes, escenario ideal para lo estigmatizantemente "delictivo". La implementación nacional y federal de los planes de viviendas que originalmente se plantearon como solución para paliar el déficit habitacional resultó, paradójicamente, deficitaria. Hoy en día, requieren de la atención y puesta en marcha de políticas articuladas para decidir cómo habitar esos espacios comunes y cómo financiar los arreglos necesarios.

En nuestro acercamiento al territorio, y por medio del mapeo de actores claves, consideramos pertinentes incorporar para nuestro análisis a la DPVyU, Unidad Ejecutora de la Ley $\mathrm{N}^{\circ}$ 12.953, Consejos de Administración, Vecinal 7 de Septiembre, Municipalidad de Rosario. El mapa debajo muestra el territorio abordado y localiza los Consejos de Administración con los que trabajamos a lo largo de nuestro análisis.

Mapa N²: Ubicación y denominación de los actores

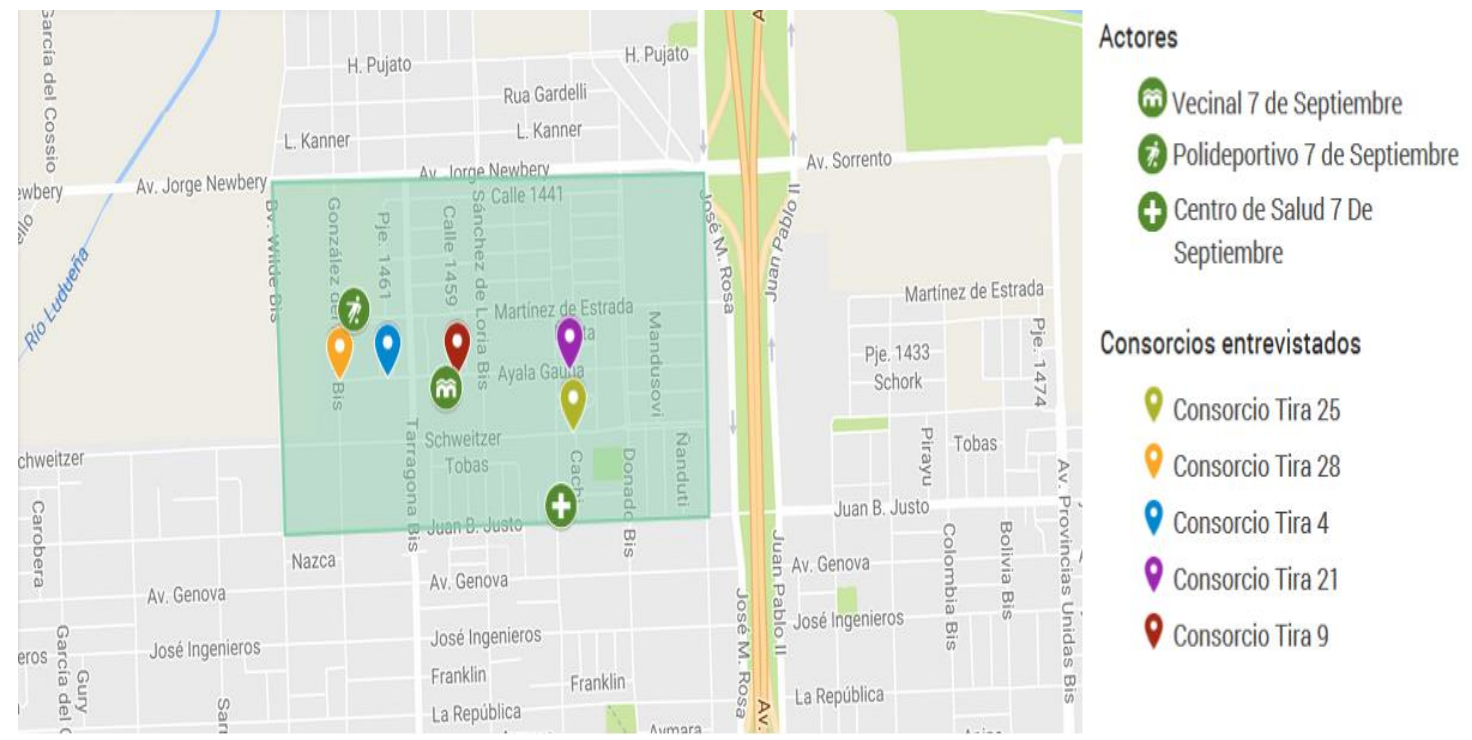

Fuente: Elaboración propia 


\section{Historicidad de la cuestión y resignificación}

La cuestión ingresó a las agendas de gestión por medio de la Ley Provincial $\mathrm{N}^{\circ}$ 12.953 del año 2008, cuya finalidad es la regularización de las situaciones jurídicas de los beneficiarios de planes ejecutados por la DPVyU para su posterior adquisición del dominio pleno mediante el otorgamiento de la escritura de las viviendas. En dicha legislación se adjunta un artículo en el que se propone concretar acciones orientadas a la constitución y capacitación de los consorcios en los Complejos Habitacionales, constituyéndose así en el primer antecedente de carácter técnico respecto al fomento de la autogestión de los habitantes.

En el mismo año se crea la Unidad Ejecutora de Titularización Ley N 12.953 para dar cumplimiento a la misma. Será a partir de allí la encargada del relevamiento de información para iniciar trámites de regularización y escrituración, así como también de la conformación y capacitación de Consejos de Administración. En consecuencia, es el actor encargado de llevar a cabo las "asambleas constitutivas" en las distintas tiras y monoblocks del barrio.

Unidad Ejecutora de Titularización se puso en funcionamiento en la delegación Sur de la Provincia de Santa Fe tras la contratación de tres profesionales abogados quienes se encargarían en adelante de realizar las actividades relacionadas con la política aquí analizada. Dada la escasa estructura asignada en una primera instancia a la Unidad Ejecutora y la mención marginal de la conformación de Consejos de Administración en la legislación, entendemos que la misma no constituía un aspecto relevante para la gestión socialista en este primer momento. De hecho, en el discurso de los técnicos de la Unidad Ejecutora, pudimos entrever que originariamente la conformación de Consejos de Administración refería en mayor medida a una tarea técnica para dar cumplimiento a una legislación que intentaba regularizar la situación de las viviendas sociales. No se consideró en ese momento como una política mediática a la cual hacerle difusión, ni se encontraron datos numéricos de la cantidad de consorcios que se generaron en el interregno 20082013. Este último año es el que marca el ingreso de esta política al Plan Abre.

En diciembre de 2011 se dio un paso importante en lo que atañe a la política habitacional con la creación de la Secretaria de Estado del Hábitat (de ahora en adelante -SEH-) de la Provincia de Santa Fe. Según palabras de la propia gestión, la nueva secretaría está orientada a promover una política habitacional más abarcativa, integradora, inclusiva y participativa. Entendemos que la creación de la secretaría da cuenta de que la cuestión comienza a adquirir mayor relevancia en la agenda gubernamental. A partir de la creación de la SEH se cambia el paradigma tecnicista, por una concepción más integral. Desde esta última perspectiva, la vivienda no sólo es entendida como un derecho sino que, además, como una base sobre la cual se hace posible estructurar otras cuestiones: las mejoras en la infraestructura o en los espacios públicos comunes pasan a ser entendidos como lugares que posibilitan mejores convivencias y relacionamientos sociales amenos. En este sentido, la problemática de la vivienda es atendida en forma instrumental para trabajar otras cuestiones. Por otra parte, el discurso gubernamental propone un enfoque participativo, abogando por la participación del ciudadano en la definición de las 
soluciones que el estado intenta brindar en materia de vivienda y promoviendo el esfuerzo compartido de vastos actores sociales (públicos y privados) en el abordaje de los problemas.

Este cambio de paradigma se da en un contexto de crecimiento del narcotráfico, del delito y de la violencia en la provincia de Santa Fe y con particular foco en la ciudad de Rosario. Esto comienza a constituirse en un tema de agenda pública y en una de las principales demandas que enfrentan los gobiernos provincial y municipal. La situación llegó a un punto cúlmine en 2013, año en donde se contabilizó el número más alto de muertes cada 100.000 habitantes, siendo los complejos habitacionales Fo.Na.Vi. el nudo de duros enfrentamientos por el reparto de zonas para la comercialización de drogas ilegales. Es imposible no recordar el caso donde acribillaron con diez balazos un departamento y luego una patrulla al interior del complejo habitacional de Grandoli al 5000 (La Capital, 2013).

Como respuesta, vemos que la gestión provincial ha comenzado a pensar sus políticas públicas desde la perspectiva de la convivencia como marco general, sobre el supuesto de que una sociedad con buenos lazos sociales, de respeto y solidaridad entre vecinos, abre a la posibilidad de pensar una sociedad más integrada, más inclusiva, con mejor calidad de vida. Para entender la racionalidad del Estado Provincial, el cual se encuentra en consonancia con el Estado Municipal debido a que ambas gestiones pertenecen al mismo color político-, es importante rescatar lo planteado en el informe de avances sobre el Plan Estratégico Provincial (2008-2015):

\begin{abstract}
"Mejorar la convivencia y recuperar los valores que nos permiten a los ciudadanos vivir en comunidad es la idea que atraviesa todas nuestras acciones y se convierte en el gran objetivo de nuestra gestión. Desde cada área de gobierno trabajamos por la convivencia social pacífica, ya que creemos que sólo una sociedad en la que se respete al de al lado, es capaz de prosperar en todas sus dimensiones: económicas, culturales, políticas y sociales."
\end{abstract}

Notamos un fuerte interés en manifestar la importancia de la convivencia como línea ejecutora a todas las políticas públicas. Un ejemplo concreto de una política pública que se pone en marcha bajo esta perspectiva es el Plan Abre, orientado a mejorar la calidad de vida en los barrios más vulnerables de las ciudades de Rosario, Santa Fe y Villa Gobernador Gálvez. La propuesta es integrar el abordaje estructural (mediante la generación de obras de infraestructura estratégicas y vinculadas a la mejora del hábitat, el equipamiento barrial, el saneamiento y al acceso a la luz y al agua) con el abordaje social (fortaleciendo las redes del barrio, promoviendo el encuentro, la participación y la convivencia en el espacio público).

Dado que la conformación de Consejos de Administración se vincula con la política habitacional, esta política fue incluida dentro del eje "Infraestructura y Hábitat" del Plan Abre, adquiriendo así un renovado impulso y un marco más integral y coordinado para su puesta en marcha. El Plan Abre creó nuevas estructuras de 
trabajo en territorio, consolidando un equipo multinivel específicamente orientado al trabajo con los consorcios de Complejos Habitacionales Fo.Na.Vi., lo que redundó en una mayor coordinación de los niveles en la implementación de la política. En otro orden de cuestiones, la incorporación de esta política al Plan Abre implicó una resignificación de la conformación de Consejos de Administración, desde entonces vinculada al impulso por mejorar la convivencia.

\section{La política pública en acción}

Abordando el territorio y realizando entrevistas a referentes de los Consejos de Administración, encontramos algunas cuestiones interesantes para visualizar el por qué la problemática cobró importancia para el gobierno provincial y para explicar por qué, posteriormente, se decide su incorporación dentro del Plan Abre. Se trata de edificios que desde su creación contaron con gran cantidad de carencias, desde la falta de servicios, la fragmentación urbana y las deficiencias estructurales. A las condiciones deficitarias en que se entregaron las viviendas, se anexaron problemas generados por falta de mantenimiento de los vecinos/consorcios, desembocando en los grandes problemas que decidimos explayar con los comentarios durante el verano de 2012 de José Peralta, representante de la Asociación Civil Barrios Fo.Na.Vi. de Rosario, quien afirmaba que:

"Las falencias estructurales son tan grandes que hay torres en este barrio y en otros que ni siquiera tienen el final de obra porque nadie quiere poner la firma ni hacerse cargo, el problema de las cloacas es histórico y uno de los más graves, además existen serios problemas de presión de agua [...] El mantenimiento de los espacios comunes, de los parques, el alumbrado en los pasillos peatonales son una pelea constante y existen usurpaciones de los propios vecinos y ajenos que construyen cocheras y ampliaciones en lugares donde no se puede". (La Capital, 2012)

En el caso del barrio 7 de Septiembre, pudimos observar problemas estructurales, de mantenimiento general, tanto en los edificios como en los espacios públicos. El déficit histórico de atención que tuvieron estos Complejos Habitacionales se refleja en el estado decadente, tanto estructural como convivencial. La situación es compleja: la mayoría de los vecinos no tiene escritura de la vivienda ni planos de la casa. Esto se traduce en un grave problema al momento de realizar obras o arreglos, ya que los vecinos desconocen los circuitos eléctricos o pluviales de las propias viviendas que habitan. A su vez, la falta de planos hace que no se pueda contar con el reglamento propio del edificio, el que indica cuáles son los espacios comunes y privados de un edificio en particular y establece las mayorías necesarias para votar en las asambleas según la importancia de cada tema, entre otras cosas. Con respecto al uso del espacio público, se observan construcciones irregulares, viéndose en cada esquina cocheras para alquilar y ampliaciones de viviendas de los vecinos de las plantas bajas. 
En otro orden de cuestiones, se trata de un barrio fuertemente estigmatizado, quizás en parte como saldo del modelo económico neoliberal implementado en la década del noventa. La política de ajuste que provocó la caída en las tasas de empleo, el aumento del empleo informal, el deterioro de los ingresos y el aumento de los trabajadores en negro, impactó claramente en las condiciones de vida de los habitantes de los monoblocks (Toppini y Uberti, 2014). Este barrio se convirtió, como gran parte de los barrios periféricos de la ciudad, en sede de economías delictivas, de hechos violentos constantes y estigmatizado de tal manera que, hasta el día de la fecha, no ingresan ambulancias sin el acompañamiento de un móvil policial.

En este contexto, la política analizada se encadena con políticas como el otorgamiento de créditos, la titularización de viviendas y las mejoras edilicias en el barrio, lo que nos genera el interrogante de saber si corresponde al gobierno provincial saldar la deuda histórica de un Estado que brindó una vivienda en condiciones deficitarias y si debe hacerse cargo de ello.

En el despliegue de la política analizada se ponen en constante tensión las responsabilidades públicas y privadas respecto a la vivienda, sus deficiencias estructurales, las faltas de obras y de mantenimiento. Si bien se trata de cuestiones que atañen a un grupo privado de personas que habitan en sus hogares, el hecho de tratarse de viviendas sociales habilita a poner en tensión estas dimensiones. Parsons (2007) nos dice que las políticas públicas se refieren a lo público y sus problemas y sostiene que el término público hace referencia a la dimensión de la actividad humana que se cree que requiere la regulación o intervención gubernamental o social, o por lo menos, la adopción de medidas comunes. En este sentido, las cuestiones que refieren a la problemática de la vivienda encuentra argumentos más sólidos que se desdibujan al momento de entender por qué la organización de los privados es el objeto de la política pública analizada. El interrogante es entonces ¿por qué el Estado Provincial debe responsabilizarse (o elige responsabilizarse) de organizar a los privados?

Tratando de dar respuesta a esta pregunta decimos que la constitución de Consejos de Administración, en conjunto con otras políticas aplicadas en el territorio, se constituye en instrumento para facilitar el abordaje de los problemas edilicios. El Estado provincial pasó a ser responsable desde la década del noventa, con el programa de descentralización, de los planes de vivienda así como del mantenimiento de las estructuras que fueron construidas previamente. Entonces, pensando en la racionalidad del Estado provincial, entendemos que se ubica desde una postura que apunta a atribuir las condiciones deficitarias de las viviendas a la falta de mantenimiento a lo largo de los años y, frente a esto, hace hincapié en la creación de Consejos de Administración como una forma para delegar sobre este nuevo actor la responsabilidad de velar por la seguridad y el mantenimiento en esos edificios.

Cabe mencionar que desde el ámbito estatal no se encuentran mayores conflictos entre los niveles y dependencias involucrados en el despliegue de la política. Principalmente debido a que las gestiones municipal y provincial (representadas en este caso por la Secretaría General, la DPVyU y la Unidad Ejecutora 
respectivamente) comparten el mismo color político, por lo que entre los mismos puede notarse un nivel de coordinación importante. También entra aquí la Vecinal del barrio puesto que, según lo hablado con el Director de la misma, la vecinal trabaja en forma articulada con la gestión municipal y con la gestión provincial. Esto quedó claramente evidenciado en las palabras del director de la Vecinal 7 de Septiembre, quien sostiene que "el estado no puede hacerse cargo de problemas internos, que son muchos y muy variados, por lo tanto debe haber alguna forma de organización que dé respuesta a los problemas que puedan surgir al interior de los Fo.Na.Vi.". Este planteo muestra una relación de afinidad con la lógica con que intervienen las dependencias estatales.

Tanto los actores provinciales como los municipales actúan sobre el supuesto de que la organización de los vecinos en consorcios por medio de la conformación de Consejos de Administración puede servir como base sobre la cual mejorar las relaciones de convivencia entre los vecinos y de ellos con el barrio en general. En palabras de técnicos de la Unidad Ejecutora se concibe la conformación de Consejos de Administración y la organización de vecinos como una manera de mejorar las condiciones de vida de los mismos que desemboca en una alternativa para reducir la franja delictiva de los barrios y, a su vez, prepara el terreno para una mejor implementación de otras políticas públicas.

Un claro ejemplo de esa lógica son las intervenciones enmarcadas en el Programa de Esfuerzo Compartido para el Mejoramiento Barrial que se realizaron en este barrio, tales como las obras de pintura y de techado. El programa sólo se puso en marcha en aquellas tiras en que los Consejos de Administración ya estuvieran legalmente conformados, por lo que se supuso la previa intervención en el territorio de la Unidad Ejecutora de la Provincia. Luego se pactaron arreglos entre la Municipalidad y cada Consejo de Administración para afrontar los costos de las obras concretadas. En el caso de la obra de techado de la Tira 9, José Villabrille, tesorero del Consejo de Administración, nos comenta que el acuerdo fue el siguiente: los vecinos compraron los materiales y la Municipalidad se encargó de obtener la mano de obra.

Sin embargo, esta coordinación entre distintos niveles y dependencias del Estado tiene una contraparte, ya que, debido a su trabajo conjunto suelen ser confundidos y categorizados todos bajo el mismo rótulo de "Fo.Na.Vi." por parte de los vecinos del barrio. Hacemos esta aclaración puesto que el desconocimiento de cada uno de los actores mencionados, de parte de los vecinos que conforman los Consejos de Administración, nos da la pauta de que las relaciones entre los mismos son escasas o carentes de solidez. Al momento de intervenir en el territorio las diversas instancias estatales no logran generar una identificación más allá de la carátula general de "Estado".

A la hora de realizar las entrevistas con los miembros de Consejos de Administración del territorio analizado notamos que las relaciones de afinidad comienzan a diluirse y aparecen lógicas y racionalidades en tensión. En primer lugar, en cada caso consultado, previamente a la conformación de Consejos de Administración de manera oficial con la Unidad Ejecutora de la Ley $N^{\circ} 12953$, los 
vecinos de este barrio ya se encontraban con algún grado de organización. Muchos de estos consorcios ya funcionaban de forma informal (carecían de personería jurídica, no estaban registrados y por tanto no contaban con documentación legal de su accionar), condición que habilitó situaciones irregulares de lo más diversas, desde vecinos que no pagaban su cuota hasta casos en que las personas encargadas de la administración malversaron los fondos recaudados. Por ello, la situación en los casos consultados no registró un cambio sustancial estrictamente hablando de organización de vecinos. En el territorio abordado se evidencia que el argumento de la constitución de Consejos de Administración formales para organizar a los vecinos, como base sobre la cual mejorar la convivencia, desconoce esta organización preexistente. Sólo en el caso de la Tira 4 las entrevistadas comentaron que el hecho de que personal de la DPVyU se acercara para dar legalidad a los consorcios le brindó a esta organización una mayor legitimidad frente al resto de los vecinos del complejo a la hora de tomar decisiones.

En segundo lugar, gran parte de los entrevistados cuestionaron la falta de asesoramiento y de seguimiento de los consorcios. Si bien los técnicos de la Unidad Ejecutora mencionan la realización de actividades que incluyen mediaciones en situaciones conflictivas y cursos de capacitación para consorcistas, tanto contables como técnico-jurídicos, en el caso de 7 de Septiembre los miembros de los Consejos de Administración encuestados nos mencionaron la ausencia de este tipo de apoyos. La participación de la Unidad Ejecutora en la implementación de la política se circunscribe a un primer momento de acercamiento a los vecinos y de asesoramiento legal para poder registrar y conformar el Consejo de Administración y el consorcio, con una personería jurídica y siguiendo todos los aspectos legales y administrativos de ese proceso. No obstante, luego de esa intervención, no encontramos instancias de seguimiento para verificar si el mismo mantiene sus reuniones, si se elaboran las actas como corresponde, si logran cobrar las cuotas o si pueden resolver los problemas y conflictos que van apareciendo. Si bien algunos de los miembros de los Consejos de Administración cuentan con contactos de abogados de la DPVyU para comunicarse en caso de consultas en lo concreto, eso queda supeditado a la iniciativa del Consejo de Administración y no a un seguimiento rutinario de los mismos.

Notamos también que en muchos casos los entrevistados tenían desconocimiento sobre diversas instancias donde recurrir: ya sea sobre la posibilidad de adquirir créditos de la DPVyU, para la titularización de las viviendas, o para la mediación en caso de conflictos que no puedan resolverse con la sola mediación del Consejo de Administración del consorcio. Es necesario denotar aquí un problema de comunicación de la política ya que estos son espacios que existen y funcionan, pero a los que los vecinos no acuden, ya sea por la falta de difusión o por una percepción de parte de los vecinos de que no tienen los resultados esperados. Vemos que se trata aquí de una relación no necesariamente conflictiva, pero sí débil y escasamente coordinada.

Cuando pensamos en la racionalidad con la que funcionan los Consejos de Administración se puede observar una ausencia de participación de los vecinos, 
miedo en el caso de tener que resolver planteos inter-vecinales y la necesidad de un tercero imparcial para decidir frente a cuestiones problemáticas, cuestiones que nos llevan a replantearnos algunos interrogantes respecto a los objetivos de participación ciudadana implícitos en la política. Con la creación de la SEH en 2011, y el cambio de perspectiva sobre la vivienda del gobierno provincial, el discurso gubernamental propone un enfoque participativo, abogando por la participación del ciudadano en la definición de las soluciones que el Estado intenta brindar en materia de vivienda.

Ahora bien, ¿en qué grado esta política de conformación de Consejos de Administración es abordada desde un enfoque participativo? En el caso de 7 de Septiembre, según la información recolectada a través de las entrevistas, esta no es una política formulada por la ciudadanía, puntualmente por los vecinos de viviendas Fo.Na.Vi., sino más bien es formulada e impulsada por el gobierno provincial tratando de producir un cambio ante el deterioro edilicio que estas viviendas estaban sufriendo por la falta de mantenimiento, que se suma a las malas condiciones en las cuales las mismas fueron entregadas al inicio. Sin embargo, es una política que trata de organizar y movilizar a los vecinos de los barrios Fo.Na.Vi de manera de que puedan administrar los espacios comunes, es decir, es una política que trata de involucrar a los vecinos en su despliegue, ya que sin esa participación no podría cumplir su objetivo. Entonces, ¿podría decirse que la política logra este nivel de involucramiento por parte de los vecinos?

Vemos que el Estado logra imponer la idea de que "hay que hacer consorcios", sin embargo, en muchos casos no logra mantener esta lógica más allá del momento de la asamblea constitutiva. Con esto queremos decir que el Estado llega a un edificio, realiza la asamblea a través de la cual se crea el consorcio y se designa al Consejo de Administración, pero esto no implica que los vecinos se mantengan organizados; es más, muchas veces se terminan desarticulando. Esta desarticulación posterior puede ser explicada por la falta de motivación de los vecinos del barrio, por la falta de apoyo estatal que se deja entrever en el discurso de los vecinos, o también, por la falta de colaboración intervecinal que desemboca en el agotamiento de los miembros de los Consejos.

Dicho todo esto, entendemos que esta política trata de instalar la participación ciudadana en un primer momento, pero no alcanza a cumplir con este objetivo. Esto se demuestra en el caso estudiado, donde puede observarse una falta de involucramiento de la mayor parte de los vecinos que conforman los consorcios. Encontramos aquí la paradoja de una política pública formulada y diseñada por actores estatales que presuponen la existencia de una participación activa de sus beneficiarios, y que, consecuentemente, cuenta con esta participación tanto en el momento de la implementación como en su posterior funcionamiento, convirtiendo de hecho a los beneficiarios en los sostenedores últimos del funcionamiento. Por lo tanto, la escasa motivación a participar, el desconocimiento de las instancias y las modalidades de participación, así como la desconfianza de algunos consorcios respecto a los efectos de la política ponen en cuestionamiento su efectivo funcionamiento. 


\section{Comentarios finales}

La política de conformación de Consejos de Administración ha sufrido ciertas resignificaciones en su puesta en marcha. En un primer momento se la consideró en un único apartado dentro de una Ley, y con el devenir de los años fue pensada como una política vehiculizadora de otras soluciones públicas. El discurso estatal sobre su aplicación luego del 2013, con la incorporación al Plan Abre, le puso énfasis al fomento de las relaciones vecinales, a mejorar la convivencia y así a brindar seguridad para los vecinos y poder gestionar conflictos. Más allá de estas intenciones sobre la situación de inseguridad en el barrio, así como en cada Tira que visitamos, ninguno de los entrevistados afirmó observar alguna mejora con respecto a esta cuestión. Aquí podemos ver que la intención en la programación de la política del gobierno municipal y provincial de incluir como eje la mejora de la "convivencia", que va desde tener barrios más seguros hasta poder contar con más solidaridad entre vecinos, no se percibe por parte de los habitantes de las tiras, quienes en las entrevistas realizadas manifestaron que la situación de inseguridad sigue latente.

Pensando en la resolución de conflictos de manera pacífica para fomentar una convivencia armónica al interior de las tiras, los vecinos manifiestan que les cuesta demasiado resolver problemas por las represalias, peleas y discusiones que esto pueda ocasionar. Aquí intentamos marcar un déficit de la política. La implementación de la misma no presentó mayores dificultades, dado que los intereses de los actores involucrados resultaron ser afines, y en los pocos casos de tensión y conflicto, éstos lograron ser sorteados sobreponiendo mayormente la racionalidad de los actores estatales. No obstante, sí se presentan enormes dificultades en su accionar. Luego de las entrevistas realizadas a los encargados del asesoramiento legal, notamos que este existió en el momento de la constitución y que, si bien ellos realizan asesoramiento en la oficina ante consultas puntuales de los vecinos, no hubo un acompañamiento del día a día de los Consejos de Administración, los cuales muchas veces muestran no tener conocimiento de lo que es la toma de decisiones colectivas, el armado de una asamblea, la resolución de conflictos por medios pacíficos, la posibilidad de mediaciones y desconocen a qué delegación gubernamental deben direccionar sus demandas, y más aún, muchos desconocen el hecho de que pueden recurrir a la Unidad Ejecutora para ser asesorados.

Es evidente que la organización de los consorcios y, por consiguiente, la conformación de Consejos de Administración ayuda a la organización de los vecinos. Pero el intento de favorecer la convivencia no parece ser el único objetivo del gobierno provincial; hay que sumarle el incentivo que supone para que los vecinos, una vez institucionalizados, puedan tomar crédito para obras de las cuales el Estado Provincial no puede ocuparse, ya sea por su magnitud o por sus costos. Este fin sólo ha sido afrontado por algunas tiras, ya que algunos de los representantes de los Consejos de Administración manifiestan tener miedo y desconfianza en el pago del resto de los vecinos para afrontar esa responsabilidad, así como otros desconocen del otorgamiento de dichos créditos. 
En relación con esto último, una de las principales falencias que hemos encontrado tiene que ver con la "comunicación" en el despliegue de la política. Los objetivos de la política deben ser transmitidos con claridad, coherencia y exactitud. Los vecinos entrevistados del barrio 7 de septiembre han comprendido la importancia de organizarse, pero no tienen un registro claro de qué dependencia les está proveyendo los productos de la política, al tiempo que carecen en la actualidad de las herramientas para poder hacerlo de forma efectiva.

Por otro lado, lo desarrollado en nuestro trabajo nos lleva a realizar un análisis sobre qué actor pudo sobreponer su lógica en el proceso de conformación de consejos de administración en el barrio 7 de septiembre. En cuanto a la asignación de las respectivas responsabilidades entre los actores intervinientes, puede decirse que en un inicio fue una cuestión muy disputada debido a que el Estado Provincial pretendía que los vecinos se hicieran cargo de la situación en que se encontraban las viviendas, mientras que los vecinos pretendían que sea alguna entidad estatal la que se hiciera cargo de la totalidad de los arreglos. Con la llegada del nuevo Código Civil y Comercial los consorcios reciben nuevas responsabilidades, y esto a su vez brinda una herramienta jurídica que favorece a que se sobreponga la racionalidad del Estado Provincial por sobre la de los vecinos. Por otra parte, pudimos observar que, si bien el Estado Provincial logró responsabilizar a los consorcios de la mejora y mantenimiento de los edificios, los vecinos lograron que distintas entidades estatales contribuyan económicamente a la hora de realizar las mejoras, ya sea poniendo la mano de obra, como en el caso de la Municipalidad de Rosario, u otorgando créditos subsidiados a los consorcios, como es el caso de la DPVyU.

Por último, aparece reiteradas veces la propuesta de un enfoque participativo. En el discurso de la gestión, se aboga por la participación del ciudadano en la definición de las soluciones que el estado intenta brindar en materia de vivienda. No obstante, creemos que se trata más bien de involucrar a los vecinos en los esfuerzos que dichas soluciones suponen. En este sentido, en el caso de la conformación de Consejos de Administración, vemos que se trata de una política que no encuentra su origen en una demanda de los vecinos y que los mismos no forman parte del proceso decisional sobre las formas y modos en que se opera. Su participación en la cuestión se reduce al momento posterior a la conformación de los Consejos de Administración, cuando ya está constituido como un actor formal y por lo tanto adquiere una legalidad para ingresar al juego de las políticas.

Recibido: $1 / 08 / 2018$

Aceptado: 11/12/2018

\section{Referencias bibliográficas:}

AGUILAR VILLANUEVA, L. (1993) "Estudio introductorio" en Problemas públicos y agenda de gobierno. Porrúa Grupo Editor, México, pg. 15-72 
BERMAN, P (1978). "The Study of Macro and Micro-Implementation", Public Policy, 26 (2), pp. 157-184. Retomado de: https:/les.scribd.com/document/46233494/5Berman-Micro-y-Macro-Implementacion

MARTINEZ NOGUEIRA, R. (2010) "La Coherencia y la Coordinación de las Políticas Públicas. Aspectos Conceptuales y Experiencias" en Los desafíos de la coordinación y la integralidad de las políticas y gestión pública en América Latina. Proyecto de Modernización del Estado, Jefatura de Gabinete de Ministros, Buenos Aires.

MORATA, F. (1991) "Políticas públicas y relaciones intergubernamentales". Documentación Administrativa, Madrid. Pgs. 153-166

OSZLAK, O. y O’DONNELL, G. (1976) “Estado y Políticas estatales en América Latina". Documento de Estudios CEDES, Buenos Aires.

OSZLAK, O. (2009) "Implementación Participativa de Políticas Públicas: Aportes a la construcción de un marco analítico" en Belmonte Alejandro (et. al.), Construyendo confianza. Hacia un nuevo vínculo entre Estado y Sociedad Civil, Volumen II. CIPPEC y Subsecretaría para la Reforma Institucional y Fortalecimiento de la Democracia, Jefatura de Gabinete de Ministros, Presidencia de la Nación, Buenos Aires.

PARSONS, W. (2007) Políticas Públicas. Una introducción a la teoría y la práctica del análisis de políticas públicas. Miño \& Dávila, coeditado con FLACSO-México.

Subirats, Joan (1989) "Análisis de las Políticas Públicas y Eficacia de la administración". INAP, Madrid.

TAPELLA, E. (2007) El mapeo de Actores Claves, documento de trabajo del proyecto Efectos de la biodiversidad funcional sobre procesos ecosistémicos, servicios ecosistémicos y sustentabilidad en las Américas: un abordaje interdisciplinario", Universidad Nacional de Córdoba, Inter-American Institute for Global Change Research (IAI).

TOPPINI, L. y UBERTI, O. (2014). "Proyectos utópicos, realidades distópicas. Interrogantes para pensar la rehabilitación de los complejos de vivienda social estatal Fo.Na.Vi tipo monoblock en Argentina " en "The Urban Incomplete: Conflicts, materialization and politics". Recuperado de: htttp.materialpolitics.com/urbanprovocations-?lightbox=dataltem-ivmdckcl

\section{Documentos oficiales y de gestión}

INFORME DE GESTIÓN (2011-2015) de la Municipalidad de Rosario LEY No 12.953 de la Provincia de Santa Fe

PLAN ESTRATÉGICO PROVINCIAL (2008-2015) de la Provincia de Santa Fe

RESOLUCIÓN Nº 0025 de Abril de 2013 de la Secretaría de Estado del Hábitat de la Provincia de Santa Fe - Programa Esfuerzo Compartido para el Mejoramiento Barrial

\section{Notas periodísticas}

LA CAPITAL (31 de marzo de 2012), "Los Fonavis padecen serios problemas estructurales”. Recuperado de: http://www.lacapital.com.ar/la-ciudad/los-fonavispadecen-serios-problemas-estructurales-n362392.html

LA CAPITAL (10 de julio de 2013), "Denuncias en la Justicia por violencia creciente y tiros en el barrio de Pimpi”. Recuperado de: 
http://www.lacapital.com.ar/policiales/denuncias-la-justicia-violencia-creciente-ytiros-el-barrio-pimpi-n433714.html

LA CAPITAL (13 de enero de 2014), "En Rosario se registraron en 2013 más del doble de crímenes que en Córdoba". Recuperado de: http://www.lacapital.com.ar/policiales/en-rosario-se-registraron-2013-mas-del-doblecrimenes-que-cordoba-n452558.html

ROSARIO NOTICIAS (21 de abril de 2016) "Distrito Noroeste: avanzan las obras en el barrio 7 de Septiembre" Recuperado de: http://rosarionoticias.gob.ar/page/noticias/id/50791/title/Avanzan-obras-en-el-barrio7-de-Septiembre ROSARIO NOTICIAS (23 de noviembre de 2016) "Anuncian nueva etapa de obras del Plan Abre en barrio 7 de Septiembre". Recuperado de: http://www.rosarionoticias.gob.ar/page/noticias/id/66109/title/Anuncian-nueva-etapade-obras-del-Plan-Abre-en-barrio-7-de-Septiembre-

\section{Entrevistas realizadas}

30/03/2017 Entrevista a Juan Ozuna, presidente de la Vecinal 7 de Septiembre.

30/03/2017 Entrevista a José Villabrille, tesorero del Consejo de Administración del Consorcio de la Tira 9 del Fo.Na.Vi. 7 de Septiembre.

13/04/2017 Entrevista a Ubaldo Modarelli, coordinador territorial de la Unidad Ejecutora de la Ley $\mathrm{N}^{\circ} 12953$.

20/04/2017 Entrevista a Carlos Soler, personal profesional de la Unidad Ejecutora de la Ley $\mathrm{N}^{\circ} 12953$.

03/05/2017 Entrevista a Silvia Pereyra, miembro del Consejo de Administración del Consorcio de la Tira 28 del Fo.Na.Vi. 7 de Septiembre.

05/05/2017 Entrevista a Alberto Oviedo, miembro del Consejo de Administración del Consorcio de la Tira 25 del Fo.Na.Vi. 7 de Septiembre.

05/05/2017 Entrevista a Marianela Perelli, Nelida Cejas y Yanina Benegas, miembros del Consejo de Administración del Consorcio de la Tira 4 del Fo.Na.Vi. 7 de Septiembre.

14/07/2017 Entrevista a Mariel Arauz, Secretaría General de la Municipalidad de Rosario. 